\title{
Apoptosis in Critical Illness: A Primer for the Intensivist
}

\author{
Z. Malam and J.C. Marshall
}

\section{Introduction}

The complexities of the cell cycle have occupied a prominent place in the history of cellular biology. Recognition of the process of mitosis dates back over a century, when Fol, Butschli, and Strasburger identified a network of intracellular points and lines, then called the karyokinetic figure, and today known as the mitotic apparatus. This discovery, dating to 1873 , laid the foundation for the discovery of chromosomes and, later, the fundamental biologic processes of mitosis and meiosis [1]. But, while cellular growth and proliferation were understood to be essential to the emergence of multicellular organisms, the corollary - that controlled cell death must be part of this calculus of cellular homeostasis - was not appreciated until quite recently. Although cell death was first described in 1859 by Virchow, it took more than a century to appreciate the importance of programmed cell death as a physiological process that eliminated unwanted cells [2]. The term 'apoptosis' was coined in 1972 by Kerr, Wyllie, and Currie to describe a distinct type of cell death characterized by the degradation of cellular constituents into membrane-bound apoptotic bodies [3]. Since then, recognition of the importance of apoptosis in health and disease, and an understanding of its cellular mechanisms, has increased exponentially.

\section{Cellular Mechanisms of Apoptosis}

The word 'apoptosis' is of Greek origin, derived from 'apo' referring to separation, and 'ptosis', translated as 'falling off, but more commonly used to describe the shedding of leaves from trees [2]. Apoptosis describes a discrete form of genetically programmed cell death that is central to development and homeostasis in metazoans [4], and that differs from cell death by necrosis which is precipitated by cellular damage, resulting in uncontrolled release of intracellular constituents [2]. The basic mechanisms of apoptosis are highly conserved (Fig. 1).

\section{Induction and Expression of Apoptosis}

Apoptosis results in the efficient, and non-inflammatory removal of redundant, senescent, transformed, or infected cells [5]. Central to this process is the degradation of intracellular proteins by members of a family of intracellular cysteine proteases called caspases, so named because they cleave their target proteins through the recognition of tetrapeptide sequences adjacent to an aspartic acid residue. There are at least 14 caspases in mammals, eleven of which are found in humans; 


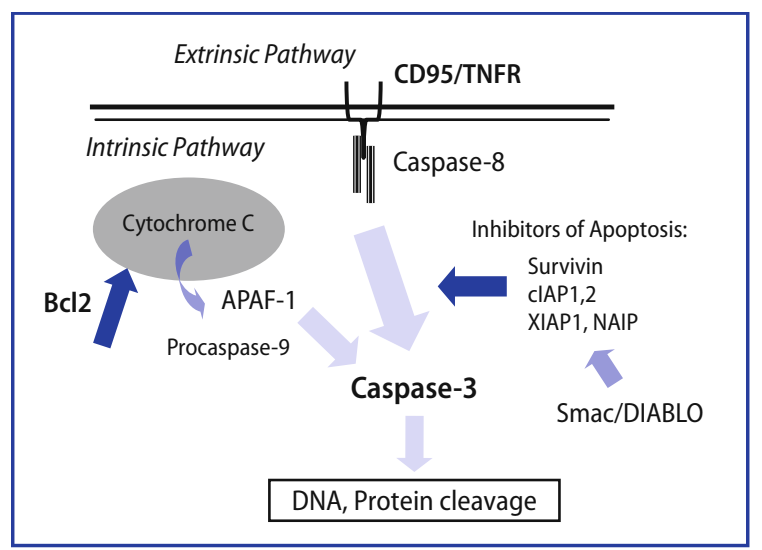

Fig. 1. Apoptosis is initiated in a receptor-mediated manner through the extrinsic pathway, or through altered mitochondrial permeability via the intrinsic pathway. Activation of either results in the assembly of macromolecular complexes that lead to initiator caspase activation, and, in turn, to the activation of effector caspases such as caspase-3. This process can be inhibited by intracellular inhibitor of apoptosis proteins, and the action of these, in turn, can be blocked by proteins such as Smac/DIABLO. See text for details.

seven of these have important established roles in apoptosis [6]. The caspase family can be further subdivided into initiator caspases that trigger the apoptotic cascade, and effector caspases responsible for enzymatic cleavage of a range of intracellular proteins, ultimately causing cell death [7]. The initiator caspases (primarily caspases- 8 and -9 , but also including caspases-2, and -10) are characterized by having at least one adaptor domain in their N-terminal region [7]. They are autoactivated and tightly regulated, through the assembly of multi-component complexes [8]. Effector caspases - predominantly caspases-3, -6, and -7 - contain prodomains that are activated by an initiator caspase through cleavage at specific aspartate residues.

Two principal pathways of apoptosis exist in mammalian cells, intrinsic and extrinsic, determined by the origin of the death stimulus. The intrinsic pathway is initiated by stimuli that originate within the cell, such as DNA damage, or that directly induce cellular damage, such as ionizing radiation, and is triggered by changes in mitochondrial permeability [7]. Alterations in mitochondrial transmembrane potential trigger the release, from the mitochondrial intermembrane space into the cytoplasm, of proteins including cytochrome C,

Smac/DIABLO, apoptosis-inducing factor (AIF), endonuclease G (EndoG), and Omi/HtrA2 [9]. The actions of the pro-apoptotic protein, cytochrome $\mathrm{C}$, are best characterized. Cytochrome $\mathrm{C}$ binds to apoptotic protease activating factor (APAF)-1 in the cytoplasm, inducing a conformational change that permits the binding of ATP/dATP to APAF-1, and recruiting pro-caspase- 9 to create a multi-protein complex termed the apoptosome. Formation of the apoptosome results in the cleavage of pro-caspase-9, releasing activated caspase- 9 , and so triggering the apoptotic process, with activation of effector caspases, such as caspase- 3 and caspase-7 [7, 10]. Other mitochondrial proteins such as Smac/DIABLO or Omi/HtrA2, released by changes in mitochondrial transmembrane potential, support the progression of apoptosis by blocking the activity of inhibitory proteins of the inhibitor of apoptosis protein (IAP) family.

The extrinsic pathway is activated by the binding of an extracellular death ligand to its cell-surface death receptor. Death ligands, such as FasL and tumor necrosis factor (TNF)- $\alpha$, interact with their receptors as homotrimers (complexes of three identical proteins), and so result in the clustering of three corresponding receptors, for example Fas (CD95) or the TNF- $\alpha$ receptor 1 (TNFR1), respectively. Each death 
receptor is a transmembrane protein that contains a cytoplasmic death domain that connects the components of the apoptotic pathway intracellularly [11]. The association of receptor death domains induces recruitment of additional adaptor proteins that also contain death domains. Activated Fas receptor recruits Fas-associated death domain (FADD), which, in addition to containing a death domain, also has a death effector domain (DED); the DED on FADD recruits and binds caspase- 8 . The resultant Fas/FADD/caspase-8 protein complex, termed the death-inducing signaling complex (DISC), leads to autocleavage and activation of caspase-8 [12], and, in turn, cleavage and activation of the effector caspase, caspase-3 [7]. TNF- $\alpha$ binding to TNFR1 also leads to receptor trimerization and the recruitment and binding of TNFR-associated DD-containing proteins (TRADDs) through their death domains. Like FADD, TRADD can recruit and activate caspase-8, activating caspase- 3 and inducing apoptosis. However, TRADD can also associate with secondary adaptor molecules that include TNFR-associated factor-2 (TRAF2) and receptor-interacting protein (RIP): This interaction results in activation of the transcription factors nuclear factor-kappa B (NF- $\mathrm{KB})$ and activator protein (AP)-1 [13, 14], that promote the transcription of IAP family members [15]. Thus, TNF- $\alpha$ can subserve the contradictory roles of inducing or inhibiting apoptosis - the actual biologic activity resulting from TNFR engagement being dependent on poorly understood accessory signals accompanying receptor engagement [11].

Caspase-3 activation, through either the intrinsic or extrinsic pathway, leads to cleavage of key cytostructural proteins, such as actin. Cleavage of another key protein target the inhibitor of caspase-activated DNase (ICAD) - results in the release of caspase activated DNase (CAD), an executioner of DNA fragmentation. Finally, the nuclear DNA repair enzyme, poly (ADP-ribose) polymerase (PARP), is inactivated through cleavage by caspase-3 [16]. Together these processes result in chromatin condensation, and the creation of membrane-bound apoptotic bodies.

The intrinsic and extrinsic pathways can interact at multiple levels. For example, activated caspase- 8 can cleave Bid, a pro-apoptotic member of the Bcl-2 protein family. This truncated form of Bid (tBid) promotes the release of cytochrome $\mathrm{C}$ from mitochondria, thus activating the intrinsic pathway of apoptosis [17].

\section{Regulation of Apoptosis}

Regulation of the intrinsic pathway occurs primarily through the Bcl-2 family of proteins. $\mathrm{Bc} 1-2$ family members share at least one Bcl-2 homology $(\mathrm{BH})$ domain, and are subdivided into proteins having pro-apoptotic or anti-apoptotic activity. Pro-apoptotic members of the Bcl-2 family, including Bax, Bad, Bak, Bid, and Bik, can be further subdivided on the basis of having only a $\mathrm{BH} 3$ domain, and so requiring assistance from pro-apoptotic members containing multiple $\mathrm{BH}$ domains in order to induce apoptosis. Pro-apoptotic Bcl-2 proteins regulate the formation of large openings in the mitochondrial outer membrane, allowing for the release of intramembrane space proteins and the induction of apoptosis [18]. Bcl-2, the initial member of this family to be identified, was isolated as a protein over-expressed in lymphocytes from a patient with a B cell lymphoma. Although the precise mechanism through which Bcl-2 inhibits apoptosis is unknown, it has been proposed that its activity arises through its capacity to block cytochrome $\mathrm{C}$ release, and to decrease $\mathrm{Ca}^{2+}$ sensitivity of the mitochondrial pore opening [19]. Other anti-apoptotic Bcl-2 members, such as Bcl-2 and Bc1-XL, may bind and sequester pro-apoptotic molecules, such as Bax, Bak, and Bid, through a hydrophobic cleft. Other anti-apo- 
ptotic proteins related to the $\mathrm{Bcl}-2$ family, including $\mathrm{Mcl}-1$ and $\mathrm{Al}$, lack a protein domain necessary for interactions with pro-apoptotic Bcl-2 family members. Mcl-1 is found primarily in hematopoietic cells and exhibits rapid turnover; thus, protection from apoptosis is short-lived [20]. Little is known about the mechanism of action of Mcl-1 or A1, or about the specific protein targets with which they interact.

The IAP family of proteins inhibits the progression of apoptosis within the cytoplasm. Originally identified as anti-apototic proteins encoded in the baculovirus genome, the IAP family is highly conserved and present in both mammals and Drosophila. There are eight mammalian IAPs: XIAP, cIAP-1, cIAP-2, ML-IAP/livin, ILP2, NAIP, Bruce/Apollon, and survivin; all but ML-IAP and ILP-2 are found in humans. Caspase-9 is inhibited primarily by XIAP, which also inhibits caspase- 3 and -7; the latter caspases may also be inhibited by cIAP-1, cIAP-2, and NAIP. All IAPs contain at least one baculoviral repeat (BIR) domain, although each BIR domain may be responsible for distinct functions. XIAP inhibits activated caspase- 9 through its third BIR domain, while caspase- 3 and -7 are targeted by a region between its first two BIR domains [7]. The mechanism of caspase-9 inhibition appears to involve heterodimerization with monomeric caspase-9, preventing apoptosome formation and caspase- 9 autoactivation [21]. Inhibition of the effector caspases-3 and -7 by XIAP occurs through a linker peptide preceding its second BIR domain. This peptide occupies the active site of caspase- 3 or -7 and serves to block substrate contact [7].

A further level of complexity in the regulation of apoptosis occurs through proteins that can block IAPs. For example, Smac/DIABLO released from mitochondria during activation of the intrinsic pathway contributes towards apoptosome formation, leading to activation of caspase-9, a process inhibited by XIAP. Binding of XIAP to caspase- 9 occurs through an IAP-binding tetrapeptide motif on caspase- 9 that allows for recruitment of XIAP and inhibition of caspase-9 activity. Smac/DIABLO possesses a similar IAP-binding tetrapeptide motif that can bind to the BIR3 domain of XIAP, and so restore caspase-9 activation [7].

\section{Dysregulated Apoptosis in the Pathogenesis of Critical IIIness}

The leading cause of death for critically ill patients in an intensive care unit (ICU) is the multiple organ dysfunction syndrome, a complex disorder that arises in association with an activated systemic inflammatory response and that has a mortality of $60 \%$ or higher when three or more organs are affected for 7 or more days [22]. The evolution of multiple organ dysfunction syndrome is associated with profound alterations in metabolic, immunologic, and cardiovascular homeostasis, and even more fundamentally, with alterations in cell survival through either excessive activation, or abnormal inhibition, of the normal kinetics of programmed cell death.

\section{Excessive Apoptosis as a Mechanism of Multiple Organ Dysfunction Syndrome}

Many of the classic cellular processes and biochemical mediators of systemic inflammatory response syndrome (SIRS)/multiple organ dysfunction syndrome have the capacity to promote apoptosis in organ and endothelial cells. Apoptosis can be accelerated, for example, by increased release of some pro- and anti-inflammatory cytokines, increased oxidant production, increased release of heat shock proteins (HSPs) from the liver, elevated glucocorticoid levels, and the release of bacterial prod- 
ucts into the circulation. Independently, these factors can alter apoptosis, however their aggregate effect in the setting of critical care may amplify this potential [23].

Vascular endothelial damage is a prominent feature of early organ dysfunction [24]. Recruitment of activated neutrophils may contribute to endothelial injury through the action of neutrophil-derived reactive oxygen species that have been implicated in altering apoptosis signaling pathways. An in vitro model of endothelial cell injury using porcine aortic endothelial cells showed that these cells underwent apoptosis when exposure to lipopolysaccharide (LPS) or cytokines was followed by heat shock; independently, these stimuli did not precipitate apoptosis. When antioxidants were administered prior to initial LPS stimulation, apoptosis was inhibited [23]. Apoptotic endothelial cell damage can potentially mediate organ injury by impeding normal tissue oxygen delivery.

Other cell populations manifest increased rates of apoptosis in pre-clinical models of critical illness. Hotchkiss et al. showed increased rates of lymphocyte apoptosis in spleen, thymus, ileum, colon, lung, and skeletal muscle in a murine model of sepsis; parenchymal cells in ileum, colon, lung, kidney, and skeletal muscle were also shown to be apoptotic [25]. Differing cell populations show variable susceptibility to apoptotic stimuli. For example, aortic endothelial cells demonstrate greater susceptibility to endotoxin-induced apoptosis than pulmonary artery, and left and right ventricular endothelial cells [26].

The acute respiratory distress syndrome (ARDS) is a common complication of critical illness. Bronchoalveolar lavage (BAL) fluid from ARDS patients can induce both fibroblast and endothelial cell apoptosis, suggesting the presence of pro-apoptotic factors in the BAL fluid. Furthermore, histological analysis of lung tissue in ARDS revealed apoptotic cell death in airspace granulation tissue [27].

The liver is particularly susceptible to endotoxin-induced apoptotic injury, perhaps because it contains an increased number of TNF receptors and inflammatory cells capable of producing inflammatory mediators [26]. Increased levels of transaminases and hepatocyte apoptosis are observed following TNF or LPS administration in a murine model; anti-TNF therapy blocked both phenomena, suggesting that they are mediated by TNF [28]. In a porcine model, endotoxin administration increased plasma levels of TNF, upregulated inducible nitric oxide synthase (iNOS), and decreased anti-apoptotic Bcl-2 levels in liver and spleen [26]. On the other hand, liver apoptosis was not seen in the cecal ligation and puncture (CLP) model reported by Hotchkiss [25].

Apoptosis has also been observed in the kidney in models of acute illness. In vivo studies show apoptotic death of renal tubular cells during reperfusion, while in vitro studies demonstrate this effect during both hypoxia and reperfusion. Apoptosis of renal cortical cells was seen 12 hours following reperfusion in a rat model [26]. The number of apoptotic renal cells in mice correlates with prolonged renal ischemia and the time following reperfusion [23]. In vitro, apoptosis of renal tubular cells can also occur following exposure to Escherichia coli-derived toxins and verotoxins. Nephrotoxic acute renal failure can result from increased apoptosis in response to therapies administered to critically ill patients. Rats given gentamicin exhibited increased apoptosis in proximal and distal tubules, while patients who received an overdose of ciprofloxacin developed distal tubular apoptosis [26]. Work by Imai and colleagues showed that injurious mechanical ventilation strategies in rabbits evoked Fas ligand-dependent epithelial cell apoptosis in the kidney [29].

Although myocyte loss in critical illness has been attributed to necrosis, apoptotic cell death has also been implicated as a mechanism of injury. Following myocar- 
dial infarction, reduced perfusion and greater myocardial stretch can serve as triggers for apoptosis [26]. Myocardial infarction leads to myocyte apoptosis in rats, and reperfusion injury to cardiomyocyte apoptosis in rabbits [23]. Patients with end-stage congestive heart failure undergoing heart transplantation also exhibited cardiomyocyte apoptosis, and showed alterations in the expression of apoptotic mediators. Pro-apoptotic members of the Bcl-2 family remained unchanged, however anti-apoptotic members showed a 1.8 fold increase in expression when compared with levels in normal hearts; rates of apoptosis in failing hearts were 232 times greater than in normal hearts [30].

Lymphoid tissues also show evidence of aberrant apoptosis in critical illness. T-cell suppression, and a reduction in numbers of circulating T-lymphocytes are common characteristics of SIRS and multiple organ dysfunction syndrome [23]. Increased thymocyte apoptosis appears to contribute to thymic involution and T-cell suppression in a mouse model of gram-negative or-positive sepsis [31], and thymocyte apoptosis was reported in a rat CLP model. Thymocyte deprivation of interleukin (IL)-2 triggers thymocyte apoptosis, suggesting that low IL-2 levels may contribute to sepsis-associated T-cell depletion [23, 32].

Extensive lymphocyte and gastrointestinal epithelial cell apoptosis have been observed in sepsis in both animal models and human patients [25, 33, 34]. Postmortem examination of patients with sepsis revealed widespread apoptosis of lymphocytes in the spleen, intestinal lamina propria, and lymphoid aggregates. Further phenotypic studies comparing spleens from septic patients with those from critically ill patients who did not have sepsis showed that the particular lymphocytes affected include B cells, CD4+ T cells, and dendritic cells, but not macrophages $[33,34]$.

Loss of muscle mass is believed to contribute to neuromuscular weakness both during and following critical illness. Such weakness manifests clinically as hypoventilation, prolonged dependence on mechanical ventilation, delayed mobilization, and muscle contractures. Although loss of muscle mass has been ascribed to accelerated protein breakdown due to systemic catabolism, myocyte apoptosis also appears to be a contributing factor. Following burn injury, apoptosis is accelerated in muscles located both adjacent to, and distant from, the burn site [26].

Accelerated apoptosis in multiple tissue beds, therefore, appears to contribute to organ dysfunction by depleting functional cells. However, apoptotic failure has also been implicated in the pathogenesis of acute organ failure.

\section{Inadequate Apoptosis as a Mechanism of MODS}

Neutrophils are key effectors of the innate immune response to infection. Their migration to sites of infection occurs early in order to eliminate pathogens through phagocytosis and the production of toxic mediators. In the absence of pro-survival stimuli from the inflammatory microenvironment, neutrophils are constitutively apoptotic, with a circulating lifespan of 8-20 hours. However multiple stimuli - of both microbial and host origin - can subvert this process, and prolong the functional survival of the neutrophil (Table 1). The normal expression of apoptosis and clearance of neutrophils from sites of inflammation is important in minimizing inadvertent bystander injury secondary to the release of neutrophil cytotoxic intracellular contents, as occurs during death by necrosis. Neutrophil longevity thus reflects a crucial balance between survival to fight infection and timely death to prevent collateral tissue damage. Additionally, neutrophil apoptosis must be regulated 
Table 1. Factors implicated in the inhibition of neutrophil apoptosis

\begin{tabular}{ll}
\hline Microbial Products & Host-derived Mediators \\
Endotoxin & Interleukin (IL)-1 $\beta$ \\
Lipoteichoic acid & IL-2 \\
Mannan & IL-3 \\
Modulins from coagulase-negative Staphylococci & IL-4 \\
E. coli verotoxin & IL-6 \\
H. pylori surface proteins & IL-8 \\
Butyric acid & IL-15 \\
Propionic acid & Tumor necrosis factor \\
Respiratory syncytial virus & Interferons \\
& G-CSF, GM-CSF \\
Physiologic Processes & Leptin \\
Transendothelial migration & Pre-B cell colony enhancing factor \\
Hypoxia & C5a \\
Acidosis & Cathelicidins \\
& Leukotriene B4 \\
\hline
\end{tabular}

Fig. 2. Whereas neutrophils harvested from healthy volunteers undergo spontaneous apoptosis following 24 hours of in vitro culture, this process is inhibited following culture with bacterial LPS, and further suppressed in circulating neutrophils from critically ill septic patients. Adapted from [38]

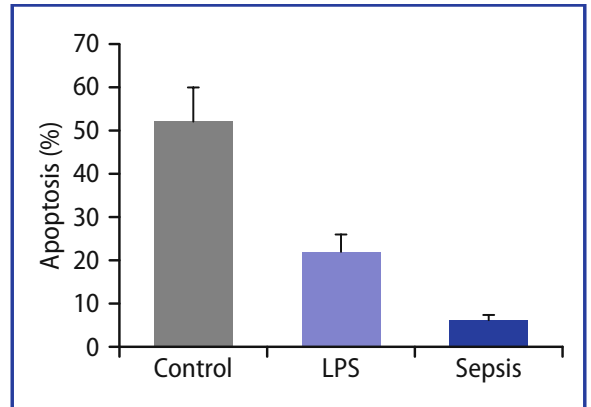

carefully in order to maintain appropriate numbers within the body, since approximately $10^{\prime \prime}$ neutrophils are produced daily by healthy adults [11, 35].

Suppressed neutrophil apoptosis has been described during a number of acute and chronic inflammatory diseases, including burn injury [36], multiple trauma [37], sepsis [38], and acute pancreatitis [39] (Fig. 2). Neutrophils harvested from BAL fluid from patients with ARDS show marked delays in apoptosis [40], and BAL fluid from patients with ARDS imparts anti-apoptotic effects on neutrophils, dependent on concentrations of granulocyte colony-stimulating factor (G-CSF) and granulocyte-macrophage colony stimulating factor (GM-CSF). Conversely, the phagocytosis of bacteria and fungi accelerates neutrophil apoptosis. In a rat model of intestinal ischemia/ reperfusion injury associated with marked pulmonary neutrophilia, intratracheal administration of killed E. coli attenuated lung injury, and improved survival [41].

\section{Therapeutic Implications}

An enhanced understanding of the mechanisms of apoptosis, and of its role in the pathogenesis of disease, has begun to define potential options for therapeutic intervention to modulate apoptosis, and to point to new mechanisms of action for established therapies. 
The anti-inflammatory activity of corticosteroids arises, in part, from their capacity to increase apoptosis in eosinophils, T-cells, and monocytes. Glucocorticoids can suppress the cell survival transcription factor, AP-1, in lymphocytes, and so inhibit the transcription of survival factors. Studies in knockout mice indicate that both APAF-1 and caspase-9 are essential for corticosteroid-induced apoptosis of thymocytes. Conversely, caspase- 1 and -3 deficiencies do not prevent corticosteroidinduced apoptosis [42]. The intracellular activities of steroids are complex, and glucocorticoids can affect the expression of the same genes in opposite ways, depending on the tissue and biologic context [43]. Glucocorticoids can also support the resolution of inflammation by enhancing the uptake of apoptotic neutrophils by phagocytic cells [44].

Significant decreases in plasma glutamine levels have been observed in critical illness, and glutamine deficiency has been associated with increased mortality in critically ill patients. Clinical trials studying glutamine administration in critically ill patients have suggested improvements in mortality, length of stay, and infectious morbidity [45]. Modulation of apoptosis is a potential mechanism for these effects. In a rat intestinal epithelial cell line, glutamine starvation for 24 hours caused a $60 \%$ reduction in cell numbers as a consequence of increased apoptosis and caspase- 3 activation. Glutamine is also required to protect human T cells from apoptosis. Glutamine downregulated Fas and FasL but upregulated Bcl-2 in stimulated T cells, raising the apoptotic threshold of these cells. Furthermore, glutamine inhibited the activation of caspase- 3 and -8 in stimulated Jurkat $T$ cells [16].

Specific caspase inhibitors have been developed and evaluated in experimental models. Mice administered a broad-spectrum caspase inhibitor, zVAD-fmk, were protected from LPS-induced acute lung injury (ALI) and its attendant lethality [46]. The use of a selective caspase- 3 inhibitor or a polycaspase inhibitor to prevent lymphocyte apoptosis in mice subjected to CLP resulted in improved survival [47]. Pretreatment with a caspase-3 inhibitor protected against TNF-mediated hepatocyte apoptosis. Additionally, caspase inhibition decreased apoptosis in models of renal, cardiac, cerebral, and hepatic ischemia/reperfusion injury, decreased infarct size in cardiac and cerebral ischemia, and improved survival in hepatic ischemia [26]. However caspase inhibition is not universally beneficial, for at least one group has shown that zVAD-fmk intensified TNF-induced toxicity by augmenting oxidative stress and mitochondrial damage, leading to increased mortality [48].

\section{Conclusion}

Apoptosis is a highly conserved physiologic process that is fundamental to the survival of multicellular organisms. Alterations in its expression leading to either excessive or deficient apoptosis are emerging as important mechanisms of disease; an evolving body of literature implicates both excessive and inadequate apoptosis in the pathogenesis of critical illness. That apoptosis is also a highly regulated process suggests the potential for interventions to taget dysregulated apoptosis, and so to restore normal kinetics of cell death, and, hopefully, attenuate acute organ injury. However the translation of this complex biology into efficacious new therapies for the critically ill remains elusive. 


\section{References}

1. Gourret JP (1995) Modelling the mitotic apparatus. From the discovery of the bipolar spindle to modern concepts. Acta Biotheor 43:127-142

2. Duque-Parra JE (2005) Note on the origin and history of the term "apoptosis". Anat RecB New Anat 283:2-4

3. Kerr JF (2002) History of the events leading to the formulation of the apoptosis concept. Toxicology $181-182: 471-474$

4. Jacobson MD, Weil M, Raff MC (1997) Programmed cell death in animal development. Cell $88: 347-354$

5. Kerr JF, Wyllie AH, Currie AR (1972) Apoptosis: a basic biological phenomenon with wideranging implications in tissue kinetics. Br J Cancer 26:239-257

6. Shi Y (2002) Mechanisms of caspase activation and inhibition during apoptosis. Mol Cell 9:459-470

7. Riedl SJ, Shi Y (2004) Molecular mechanisms of caspase regulation during apoptosis. Nat Rev Mol Cell Biol 5:897-907

8. Adams JM, Cory S (2002) Apoptosomes: engines for caspase activation. Curr Opin Cell Biol $14: 715-720$

9. Wang X (2001) The expanding role of mitochondria in apoptosis. Genes Dev 15:2922-2933

10. Yuan J, Yankner BA (2000) Apoptosis in the nervous system. Nature 407:802-809

11. Akgul C, Edwards SW (2003) Regulation of neutrophil apoptosis via death receptors. Cell Mol Life Sci 60:2402-2408

12. Medema JP, Scaffidi C, Kischkel FC, et al (1997) FLICE is activated by association with the CD95 death-inducing signaling complex (DISC). EMBO J 16:2794-2804

13. Ashkenazi A, Dixit VM (1998) Death receptors: signaling and modulation. Science 281: $1305-1308$

14. Nagata S (1997) Apoptosis by death factor. Cell 88:355-365

15. Ward C, Chilvers ER, Lawson MF, et al (1999) NF-kappaB activation is a critical regulator of human granulocyte apoptosis in vitro. J Biol Chem 274:4309-4318

16. Fuchs BC, Bode BP (2006) Stressing out over survival: glutamine as an apoptotic modulator. J Surg Res 131:26-40

17. Luo X, Budihardjo I, Zou H, Slaughter C, Wang X (1998) Bid, a Bcl2 interacting protein, mediates cytochrome $c$ release from mitochondria in response to activation of cell surface death receptors. Cell 94:481-490

18. Kuwana T, Newmeyer DD (2003) Bcl-2-family proteins and the role of mitochondria in apoptosis. Curr Opin Cell Biol 15:691-699

19. Murphy E, Imahashi K, Steenbergen C (2005) Bcl-2 regulation of mitochondrial energetics. Trends Cardiovasc Med 15:283-290

20. Edwards SW, Derouet M, Howse M, Moots RJ (2004) Regulation of neutrophil apoptosis by Mcl-1. Biochem Soc Trans 32:489-492

21. Shiozaki EN, Chai J, Rigotti DJ, et al (2003) Mechanism of XIAP-mediated inhibition ofcaspase-9. Mol Cell 11:519-527

22. Marshall JC, Cook DJ, Christou NV, Bernard GR, Sprung CL, Sibbald WJ (1995) Multiple organ dysfunction score: a reliable descriptor of a complex clinical outcome. Crit Care Med 23:1638- 1652

23. Papathanassoglou ED, Moynihan JA, Ackerman MH (2000) Does programmed cell death (apoptosis) play a role in the development of multiple organ dysfunction in critically ill patients? a review and a theoretical framework. Crit Care Med 28:537-549

24. McMillen MA, Huribal M, Sumpio B (1993) Common pathway of endothelial-leukocyte interaction in shock, ischemia, and reperfusion. Am J Surg 166:557-562

25. Hotchkiss RS, Swanson PE, Cobb JP, Jacobson A, Buchman TG, Karl IE (1997) Apoptosis in lymphoid and parenchymal cells during sepsis: findings in normal and T-and B-cell-deficient mice. Crit Care Med 25:1298-1307

26. Lydon A, Martyn JA (2003) Apoptosis in critical illness. Int Anesthesiol Clin 41:65-77

27. Polunovsky VA, Chen B, Henke C, et al (1993) Role of mesenchymal cell death in lung remodeling after injury. J Clin Invest 92:388-397

28. Leist M, Gantner F, Bohlinger I, Tiegs G, Germann PG, Wendel A (1995) Tumor necrosis fac- 
tor-induced hepatocyte apoptosis precedes liver failure in experimental murine shock models. Am J Pathol 146:1220-1234

29. Imai Y, Parodo J, Kajikawa O, et al (2003) Injurious mechanical ventilation and end-organ epithelial cell apoptosis and organ dysfunction in an experimental model of acute respiratory distress syndrome. JAMA 289:2104-2112

30. Olivetti G, Abbi R, Quaini F, et al (1997) Apoptosis in the failing human heart. N Engl J Med 336:1131-1141

31. Wang SD, Huang KJ, Lin YS, Lei HY (1994) Sepsis-induced apoptosis of the thymocytes in mice. J Immunol 152:5014-5021

32. Barke RA, Roy S, Chapin RB, Charboneau R (1994) The role of programmed cell death (apoptosis) in thymic involution following sepsis. Arch Surg 129:1256-1261

33. Hotchkiss RS, Tinsley KW, Swanson PE, et al (2001) Sepsis-induced apoptosis causes progressive profound depletion of B and CD4+ T lymphocytes in humans. J Immunol 166:6952-6963

34. Hotchkiss RS, Tinsley KW, Swanson PE, et al (2002) Depletion of dendritic cells, but not macrophages, in patients with sepsis. J Immunol 168:2493-2500

35. Simon HU (2003) Neutrophil apoptosis pathways and their modifications in inflammation. Immunol Rev 193:101-110

36. Chitnis D, Dickerson C, Munster AM, Winchurch RA (1996) Inhibition of apoptosis in polymorphonuclear neutrophils from burn patients. J Leukoc Biol 59:835-839

37. Ertel W, Keel M, Infanger M, Ungethum U, Steckholzer U, Trentz O (1998) Circulating mediators in serum of injured patients with septic complications inhibit neutrophil apoptosis through up-regulation of protein-tyrosine phosphorylation. J Trauma 44:767-775

38. Taneja R, Parodo J, Jia SH, Kapus A, Rotstein OD, Marshall JC (2004) Delayed neutrophil apoptosis in sepsis is associated with maintenance of mitochondrial transmembrane potential and reduced caspase-9 activity. Crit Care Med 32:1604-1469

39. O’Neill S, O’Neill AJ, Conroy E, Brady HR, Fitzpatrick JM, Watson RW (2000) Altered caspase expression results in delayed neutrophil apoptosis in acute pancreatitis. J Leukoc Biol 68:15-20

40. Matute-Bello G, Liles WC, Radella F $2^{\text {nd }}$ (1997) Neutrophil apoptosis in the acute respiratory distress syndrome. Am J Respir Crit Care Med 156:1969-1977

41. Sookhai S, Wang JJ, McCourt M, Kirwan W, Bouchier-Hayes D, Redmond P (2002) A novel therapeutic strategy for attenuating neutrophil-mediated lung injury in vivo. Ann Surg 235:285-291

42. Distelhorst CW (2002) Recent insights into the mechanism of glucocorticosteroid-induced apoptosis. Cell Death Differ 9:6-19

43. Amsterdam A, Sasson R (2002) The anti-inflammatory action of glucocorticoids is mediated by cell type specific regulation of apoptosis. Mol Cell Endocrinol 189:1-9

44. Liu Y, Cousin JM, Hughes J, et al (1999) Glucocorticoids promote nonphlogistic phagocytosis of apoptotic leukocytes. J Immunol 162:3639-3646

45. Kelly D, Wischmeyer PE (2003) Role of L-glutamine in critical illness: new insights. Curr Opin Clin Nutr Metab Care 6:217-222

46. Mignon A, Rouquet N, Fabre M, et al (1999) LPS challenge in D-galactosamine-sensitized mice accounts for caspase-dependent fulminant hepatitis, not for septic shock. Am J Respir Crit Care Med 159:1308-1315

47. Hotchkiss RS, Chang KC, Swanson PE, et al (2000) Caspase inhibitors improve survival in sepsis: a critical role of the lymphocyte. Nat Immunol 1:496-501

48. Cauwels A, Janssen B, Waeytens A, Cuvelier C, Brouckaert P (2003) Caspase inhibition causes hyperacute tumor necrosis factor-induced shock via oxidative stress and phospholipase A2. Nat Immunol 4:387-393 